\title{
Photothermal microscopy: a step from thermal wave visualization to spatially localized thermal analysis
}

\author{
S. GALOVIĆ, Z. STOJANOVIĆ, D. ČEVIZOVIĆ \& M. POPOVIĆ \\ Vinča Institute of Nuclear Sciences, P.O. Box 522, 11001 Belgrade, Serbia
}

Key words. Imaging, photothermal, thermal analysis.

\begin{abstract}
Summary
The calculation of photothermal response detected by a photothermal microscope is presented. By using a technique based on Green's functions and integral transforms, a model for laser-induced temperature distribution functions has been derived. The mathematical method for solution of inverse problem is proposed. It suggests that photothermal microscopy, besides imaging subsurface of solids, has the potential for quantitative thermal analysis of various samples.
\end{abstract}

\section{Introduction}

There are several thermal microscopy techniques that produce thermal contrast imaging associated with variations in the thermal properties of a sample, such as thermal conductivity, specific heat, thermal diffusivity and thermal expansion (Gmelin et al., 1998). Some of these techniques, like photothermal microscopy (PTM), use an intensity-modulated laser beam to generate time-dependent heat flow inside the sample (Rosencwaig, 1982). In PTM, the thermal waves scattered from subsurface inhomogeneities can subsequently be detected using direct or indirect measurement of resultant temperature changes of the sample surfaces. For example, it is possible to monitor infrared radiation emitted from the heated sample, or the deflection of a probe laser beam traversing the heated gaseous layer just above the sample surface (mirage effect). Interferometric detection of the thermoelastic displacement of the surface or gas-microphone photoacoustic detection could also be used. Alternatively, thermoacoustic signals generated in the bulk are detected using piezoelectric sensors. Various methods of detection of thermal waves are summarized in Cretin (1999).

A scanning mechanism incorporated in PTM enables obtaining of spatial temperature mapping and imaging of

Correspondence to: S. Galović. Tel \& fax: +381112453986; e-mail: bobagal@ vin.bg.ac.yu the lateral distribution of the optical absorption and thermal properties within a sample with a resolution that is limited by the wavelength of thermal waves. In practice, this varies from about $100 \mathrm{~nm}$ upwards, with an imaging depth typically somewhat greater (Ippolito et al., 2004).

Typical applications of PT microscopy include investigations of ceramics, laminates, compound, coated surfaces and structured semi-conductors with pattern sizes from the millimeter to the sub-micrometre range (Grice et al., 1983), detection of fatigue crack in metals or fractures in brittle solids. These techniques have also been implemented onto imaging of photo-structured polymer channel waveguides, and to image refractive index pattern in polymeric integrated optical devices (Kreiter et al., 1999). Besides, the use of PTM for visualizing the internal structure of soft biological tissues is currently attracting much more attention (Beard \& Mills, 1997). A broad range of potential applications has emerged, including detection of breast, skin and oral cancer and vascular applications such as imaging of superficial blood vessels and the characterization of arterial tissues (Hoelan et al., 1998). A key advantage of this technique is that it exploits the strong optical contrast of tissues, enabling differentiation of anatomical features that would be indistinguishable using other radiological modalities.

In previous employment, the final goal of using PTM was to obtain information about the location and the shape of inhomogeneities from recorded images. In addition, this microscopic technique could be used for spatially localized thermal analysis. It requires both the detailed theoretical treatment of the physical mechanism of the PTM signal formation and the development of mathematical technique for reconstruction of thermal and optical properties from PTM images corresponding to the solution of the inverse problem.

In this paper, a detailed theoretical description of the thermal wave propagation in the three dimensions is presented. By using a technique based on Green's function and integral transformations, the model for laser-induced temperature distribution functions is derived. The mathematical method for solving the inverse problem is proposed. 


\section{Theoretical consideration}

Thermal wave propagation across the environment and the sample irradiated by a modulated laser beam perpendicular to the sample surface has been mathematically described in cylindrical geometry by a set of hyperbolic partial differential equations of heat conductions (Joseph \& Preziosi, 1989; Galovic \& Kostoski, 2003):

$$
\begin{aligned}
& \frac{\partial^{2} \vartheta_{i}}{\partial r^{2}}+\frac{1}{r} \frac{\partial \vartheta_{i}}{\partial r}+\frac{\partial^{2} \vartheta_{i}}{\partial z^{2}} \\
&=\frac{1}{D_{T i}}\left[\frac{\partial \vartheta_{i}}{\partial t}+\frac{1}{\tau_{i}} \frac{\partial^{2} \vartheta_{i}}{\partial t^{2}}\right]-\frac{S_{i} e^{\gamma z}}{k_{i}}\left[f(t)+\tau_{i} \frac{d f(t)}{d t}\right] \\
& q_{i}+\tau_{i} \frac{\partial q_{i}}{\partial t}=-k_{i} \nabla \vartheta_{i},
\end{aligned}
$$

where $i=\{a($ air $), \quad s$ (sample), $b$ (backing) $\} ; \vartheta_{i}$, dynamic temperature; $q_{i}$, heat flux; and $D_{T i}, \tau_{i}$ and $k_{i}$ are, respectively, the thermal diffusivity, thermal relaxation time and thermal conductivity of the ith area; $\gamma$ is the optical absorption coefficient of the sample and $f(t)$ denotes time-dependent modulation function of laser beam intensity. The volumetric rate of heat generation $S_{i}$ is given by

$$
S_{i}=\left\{\begin{array}{cl}
0 & i=a, b \\
A(r)=\frac{\gamma P \eta}{\pi a^{2}} \exp \left(-\frac{2 r^{2}}{a^{2}}\right) & i=s
\end{array} .\right.
$$

Here, $P$ is the power of laser beam, $\eta$ is radiation-to-heat conversion efficiency and $a$ is the $1 / e^{2}$ radius of the Gaussian pump beam.

For the sake of simplicity, it is assumed that all three regions - air, sample and backing - extend to infinity in the radial direction. The temperature and heat flux must be continuous across the area boundaries and the temperature vanishes far from the sample. The initial conditions are zero.

The problem of photothermal wave propagation described by partial differential equations (Eqs (1)-(3)) with the mentioned boundary and zero initial conditions have been reduced to the system of ordinary differential equations by both Hankel and Laplace transforms. Symbols $\lambda$ and $p$ denote integration variables in the Hankel and Laplace transforms, respectively.

The reduced set of differential equations was solved for $f(t)=\delta(t)$, that is the Green functions method is applied (Rickayzen, 1980). The general solutions for the Green functions $G_{i} i=a, s, b$ in the complex space $(\lambda, p)$ can be written as:

$$
\begin{aligned}
G_{a}(\lambda, z, p)= & A_{1}(\lambda, p) \exp \left(-\Xi_{a} z\right) \\
G_{s}(\lambda, z, p)= & A_{2}(\lambda, p) \exp \left(\Xi_{a} z\right) \\
& +A_{3}(\lambda, p) \exp \left(-\Xi_{a} z\right)-K(\lambda, p) \exp (\gamma z) \\
G_{b}(\lambda, z, p)= & A_{4}(\lambda, p) \exp \left(\Xi_{a}(z+l)\right),
\end{aligned}
$$

where

$$
\begin{aligned}
& K(\lambda, p)=\frac{A(\lambda)}{\gamma^{2}-\Xi_{s}^{2}} \\
& \Xi_{i}(\lambda, p)=\sqrt{\lambda^{2}+\frac{p\left(1+\tau_{i} p\right)}{D_{T i}}}, \quad i=a, s, b .
\end{aligned}
$$

The coefficients $A_{1}-A_{4}$ were determined by using the boundary conditions:

$$
A_{2}(\lambda, p)=\frac{\left(1+r_{b}\right)\left(r+r_{a}\right) e^{\Xi_{s} l}-\left(r-r_{b}\right)\left(1-r_{a}\right) e^{-\gamma l}}{\left(1+r_{b}\right)\left(1+r_{a}\right) e^{\Xi_{s} l}-\left(1-r_{b}\right)\left(1-r_{a}\right) e^{-\Xi_{s} l}} K(\lambda, p)
$$

$A_{3}(\lambda, p)=\frac{\left(r_{b}-r\right)\left(1+r_{a}\right) e^{-\gamma l}-\left(1-r_{b}\right)\left(r+r_{a}\right) e^{-\Xi_{s} l}}{\left(1+r_{b}\right)\left(1+r_{a}\right) e^{\Xi_{s} l}-\left(1-r_{b}\right)\left(1-r_{a}\right) e^{-\Xi_{s} l}} K(\lambda, p)$

$$
A_{1}(\lambda, p)=A_{2}(\lambda, p)+A_{3}(\lambda, p)-K(\lambda, p)
$$

$$
A_{4}(\lambda, p)=A_{2}(\lambda, p) e^{-\Xi_{s} l}+A_{3}(\lambda, p) e^{\Xi_{s} l}-K(\lambda, p) e^{-\gamma l},
$$

where $r_{i}=Z_{c s} / Z_{c i}, i=a, b, Z_{c i}=\left(1+\tau_{i} p\right) / k_{i} \Xi_{i}, i=a, s, b$ and $r=\gamma / \Xi_{s}$.

For any type of modulation, the temperature distributions could be obtained from the known Green functions (Rickayzen, 1980).

$$
\begin{aligned}
& \vartheta_{i}(\lambda, z, t) \\
& =\int_{0}^{t} f(u) G_{i}(\lambda, z, t-u) d u=\int_{0}^{t} G_{i}(\lambda, z, u) f(t-u) d u,
\end{aligned}
$$

where $G_{i}(\lambda, z, t)$ is inverse Laplace transform of Green functions given by Eqs (4)-(6):

$$
G_{i}(\lambda, z, t)=\frac{1}{2 \pi i} \int_{s-i \omega}^{s+i \omega} G_{i}(\lambda, z, p) \exp (p t) d t .
$$

Finally, the temperature distributions for different regions are obtained by taking the inverse Hankel transform of Eq. (13):

$$
\vartheta_{i}(r, z, t)=\int_{0}^{\infty} \vartheta_{i}(\lambda, z, t) J_{0}(\lambda r) \lambda d \lambda
$$

where $J_{0}(\lambda r)$ is the zero-order Bessel function of the first kind.

Surface temperature variations in $(r, t)$ domain, which are proportional to photothermal responses, could be obtained from Eqs. (4) and (13)-(15):

$$
\vartheta_{s}(\lambda, 0, t)=\int_{0}^{t}\left[\frac{1}{2 \pi i} \int_{s-i \infty}^{s+i \infty} A_{1}(\lambda, 0, p) \exp (p u) d p\right] f(t-u) d u
$$




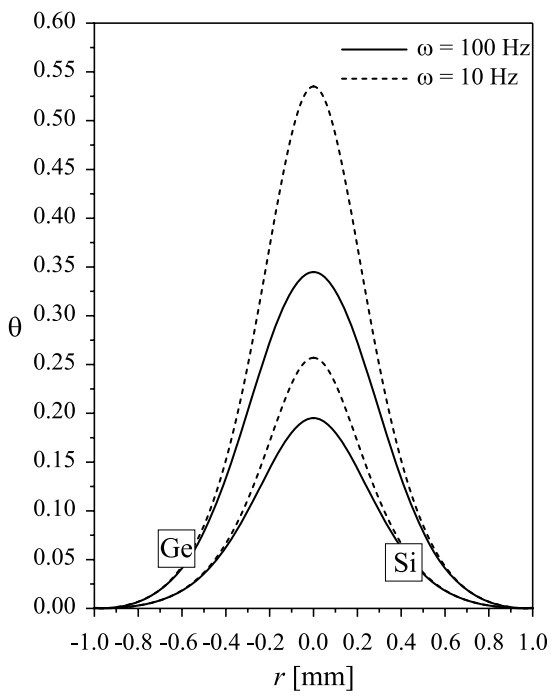

a)

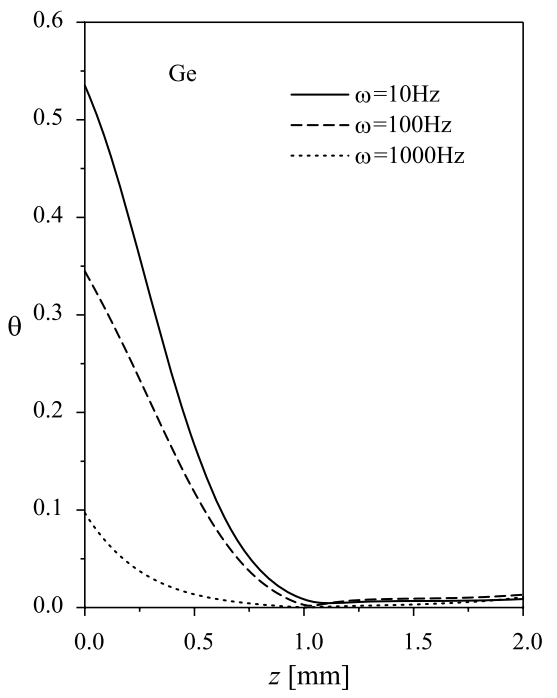

b)

Fig. 1. The temperature distribution (a) in radial direction for axial coordinate $z=0$, for two modulation frequencies, $10 \mathrm{and} 100 \mathrm{~Hz}$, and for two different media, Si and Ge; (b) in axial direction within Ge sample for radial coordinate $r=0$ and for three different modulation frequencies, 10,100 and $1000 \mathrm{~Hz}$.

$$
\vartheta_{s}(r, 0, t)=\int_{0}^{\infty} \vartheta_{s}(\lambda, 0, t) J_{0}(\lambda r) \lambda d \lambda .
$$

\section{Results and discussion}

The derived model shows that thermal properties of both the sample and environment affect the PT response, meaning that the information of all these properties is contained in the recorded image. But, as it could be concluded from the model, the influence of the sample thermal and optical properties on the PTM response is very complex. In addition, the temperature variations on the sample surfaces, Eqs. (16), (17), could not be expressed in a closed form, and they should be evaluated numerically in order to analyze the influence of thermal and optical properties of the sample on the PTM signal.

In the numerical evaluation, it is assumed that the incident beam is harmonically modulated. Surface temperature variations were calculated for various semi-conductor samples ( $\mathrm{Si}, \mathrm{Ge})$ and for various values of modulation frequency. The following air and backing thermal properties are used: $k_{a}=$ $0.025 \mathrm{WmK}^{-1}, D_{T a}=1938 \times 10^{-8} \mathrm{~m}^{2} \mathrm{~s}^{-1}, k_{b}=30 \mathrm{WmK}^{-1}$, $D_{T b}=3.413 \times 10^{-8} \mathrm{~m}^{2} \mathrm{~s}^{-1}$.

As regards the local thermal analyses, the most interesting result is the following: the heat does not propagate very much beyond the extent of the pump beam in the $x-y$ plane if the thermal diffusive length is smaller than the radius of the laser beam (Fig. 1(a)). Its value depends on modulation frequency (Smallwood et al., 2002). If the modulation frequency increases, the thermal diffusive length decreases. It means that in a high-frequency range, optically induced heat flow could be considered as one-dimensional. In that case, inverse photothermal problem becomes solvable. A few algorithms could be found in the literature (Rosencwaig \& Gersho, 1976; Lan et al., 1995; Aleshin \& Vysloukh, 1997).

The shifting of the modulation frequency upwards is convenient not only for localized thermal investigations of the sample but also for the increasing of the spatial resolution of PTM and improving of the signal-to-noise ratio in the measured response. However, the larger the modulation frequency, the smaller the depth $z$ in which thermal waves are not significantly damped (Fig. 1(b)). Besides, our evaluations also show that the measured signal decreases if the modulation frequency increases (Fig. 1(a)). Therefore, the choice of modulation frequency is limited by both the values of surface thermal changes that could be measured and the maximum desired depth of the sample that is imaged.

\section{Conclusion}

The calculations of surface temperature variations proportional to the responses detected by a PT microscope are presented. The derived model shows that the information about the values of local thermal and optical properties of both the sample and the backing are contained in the PT micrograph, but quantitative determination of these properties is achievable in a high frequency range. The frequency range that is needed for solving onedimensional inverse problem could be undesirable as far as the measurement of temperature changes and imaging depth 
in some material systems is concerned. In these cases, further investigations are necessary. Nevertheless, our results suggest that besides imaging the subsurface of solids, PT microscopy has a potential for quantitative thermal analysis of various samples.

\section{Acknowledgement}

This work was supported by Ministry of Science of the Republic of Serbia (project no. 141013).

\section{References}

Aleshin, V.V. \& Vysloukh, V.A. (1997) Continued fraction method in inverse problem of photothermal diagnostic. Appl. Phys. A 64, 579582.

Beard, P.C. \& Mills, T.N. (1997) Characterization of post mortem arterial tissue using time-resolved photoacoustic spectroscopy at 436, 461 and 532 nm. Phys. Med. Biol. 42, 177-198.

Cretin, B. (1999) Multi-acquisition photothermoelastic imaging with optical probing: which image for which application. Photoacoustic and Photothermal Phenomena (ed. by F. Scudieri \& M. Bertolotti), pp. 147151. AIP, Woodbury, New York.

Galovic, S. \& Kostoski, D. (2003) Photothermal wave propagation in media with thermal memory. J. Appl. Phys. 93, 3063-3070.
Gmelin, E., Fischer, R. \& Stitzinger, R. (1998) Sub-micrometer thermal physics - an overview on SThM techniques. Thermochim. Acta 310, $1-17$.

Grice, K.R., Inglehart, L.J., Favro, L.D., Kuo, P.K. \& Thomas, R.L. (1983) Thermal-wave imaging of closed cracks in opaque solids. J. Appl. Phys. 54, 6245-6255.

Hoelen, G.G., de Mul, F.F.M., Pougers, R. \& Dekker, A. (1998) Threedimensional photoacoustic imaging of blood vessels in tissue. Opt. Lett. 23, 648-650.

Ippolito, S.B., Thorne, S.A., Fraslan, M.G., Goldberg, B.B. \& Uulu, M.S. (2004) High spatial resolution subsurface thermal emission microscopy. Appl. Phys. Lett. 84, 4529-4531.

Joseph, D.D. \& Preziosi, L. (1989) Heat waves. Rev. Mod. Phys. 61, 41-70. Kreiter, M.J.S. \& Mittler-Neher, S. (1999) Scanning photothermal beam deflection- and scanning photothermal displacement-imaging of polymer channel waveguides: a comparison. Thin Solid Films 342, 244-248.

Lan, T.T.N., Seidal, U. \& Walther, H.G. (1995) Theory of microstructure depth profiling by photothermal measurement. J. Appl. Phys. 77, 47394745 .

Rickayzen, G. (1980) Green's Functions and Condensed Matter. Academic Press, London.

Rosencwaig, A. \& Gersho, A. (1976) Theory of the photoacoustic effects with solids. J. Appl. Phys. 47, 64-69.

Rosencwaig, A. (1982) Thermal-wave imaging. Science 218, 223-228.

Smallwood, R., Metherall, P., Hose, D., et al. (2002) Tomographic imaging and scanning thermal microscopy: thermal impedance tomography. Thermochim. Acta 385, 19-32. 